\title{
Problemas diagnósticos en la enfermedad celiaca del adulto
}

\author{
L. I. Fernández Salazar, N. de la Torre Ferrera ${ }^{1}$, B. Velayos Jiménez, M. Nocito Colón² J. M. González \\ Hernández y J. A. Garrote Adrados ${ }^{3}$
}

Servicios de Aparato Digestivo y ${ }^{1}$ Medicina Interna.${ }^{2}$ Sección de Inmunología. ${ }^{3}$ Unidad de Investigación. Laboratorio de Inmunidad de las mucosas, IBGM. Hospital Clínico Universitario de Valladolid. Universidad de Valladolid

\section{RESUMEN}

Introducción: la enfermedad celiaca (EC) es una enteropatía crónica de base inmune debida a una intolerancia al gluten en sujetos genéticamente predispuestos.

Objetivos: a) describir clínica, enfermedades asociadas y serología en la EC del adulto; y b) estudiar la utilidad de la serología en el diagnóstico y su relación con la expresión clínica e histológica.

Pacientes y métodos: se han estudiado de forma retrospectiva 31 pacientes adultos con diagnóstico de EC seguidos en consulta. Se recogieron datos referidos a los síntomas de presentación, enfermedades asociadas, bioquímica, serología (anticuerpos antigliadina y AEm) y genética (HLA DQ2). Se comprobó si la clínica típica o la positividad de AEm se asociaban a diferencias clínicas, serológicas o grado de atrofia vellositaria.

Resultados: prácticamente el $50 \%$ de los pacientes tuvo manifestaciones clínicas atípicas y el $33 \%$ no tuvo síntomas gastrointestinales. La clínica típica se asoció a atrofia de vellosidades grado III b-c de Marsh (87 vs. 53\%, p = 0,03). El 70\% de los pacientes tuvo anticuerpos AEm positivos. Entre los pacientes con AEm fueron más frecuentes las mujeres $(78 v$ s. $37 \%, p=0,03)$ y la atrofia de vellosidades grado III b-c de Marsh (84 vs. 50\%, $\mathrm{p}=0,05)$. En el estudio genético, el 68,4\% (13/19) eran portadores de los dos alelos.

Conclusiones: la clínica de la EC del adulto es muy variable. La frecuencia que encontramos de AEm y genética (DQ2) es menor a la publicada. Clínica, grado de atrofia y serología podrían interrelacionarse. La genética puede complementar a los AEm en el diagnóstico.

Palabras clave: Enfermedad celiaca. Enteropatía por gluten. Enteropatía sensible al gluten. Enfermedades autoinmunes.

\begin{abstract}
Introduction: celiac disease (CD) is a chronic immune-mediated enteropathy, resulting from a gluten intolerance in genetically predisposed individuals.

Objetive: a) to describe clinical features, associated disorders and serology of CD in adults; and b) to study the main that serology displays in diagnosis, clinical and histological expression.

Patients and methods: 31 patients diagnosed of $C D$ in adulthood have been reviewed retrospectively, including clinical presentation, associated disorders, biochemical results, serological tests (anti-gliadin and anti-endomysial antibodies) and genetical features (HLA-DQ2). It has been studied the relation between typical presentations and AEm with clinical, serological or histological findings.

Results: almost $50 \%$ of patients had atypical clinical manifestations and gastrointestinal symptoms were absent in 33\%. Typical manifestations are associated with villous atrophy stage III b-c of Marsh's classification (87 vs. $53 \%, p=0,03$ ). $70 \%$ of patients shows AEm mostly in women (78 vs. $37 \%, p=0,03)$ and stage III b-c of Marsh (84 us. 50\%, p = 0,05). 68,4\% were DQ2 positive.

Conclusions: clinical features of $\mathrm{CD}$ varies greatly. AEm and DQ2 are less common than others studies. There may be an association with clinical and serological findings and villous atrophy stage. Genetical features could help AEm in diagnosis.
\end{abstract}

Key words: Celiac disease. Gluten enteropathy. Gluten-sensitive enteropathy. Autoimmune disorders.

Fernández Salazar LI, de la Torre Ferrera N, Velayos Jiménez B, Nocito Colón M, González Hernández JM, Garrote Adrados JA. Problemas diagnósticos en la enfermedad celiaca del adulto. Rev Esp Enferm Dig 2008; 100: 24-28.

Recibido: 09-10-07.

Aceptado: 15-11-07.

Correspondencia: L. I. Fernández Salazar. C/ Gamazo, 4, $3^{\circ}$ B. 47004 Valladolid.e-mail: luisfersal@wanadoo.es

\section{INTRODUCCIÓN}

La enfermedad celiaca (EC) es una enteropatía crónica debida a una intolerancia permanente a proteínas del gluten de determinados cereales en sujetos genéticamente predispuestos. En su etiopatogenia se implican factores 
ambientales, factores genéticos, por existir una tendencia a la agrupación familiar y una asociación con los alelos que codifican el DQ2 y DQ8 del HLA de clase II, y factores inmunológicos, por la presencia de anticuerpos (antiendomisio, antigliadina y antitransglutaminasa tisular) y de un infiltrado inflamatorio activo en la lámina propia intestinal $(1,2)$.

\section{OBJETIVOS}

1. Describir las manifestaciones clínicas, las enfermedades asociadas y las características serológicas en la enfermedad celiaca del adulto.

2a. Estudiar la utilidad de la serología, concretamente los anticuerpos antiendomisio (AEm), en el diagnóstico.

2b. Describir posibles relaciones de la serología y la expresión clínica e histológica.

\section{PACIENTES Y MÉTODOS}

Hemos estudiado de forma retrospectiva a los 31 pacientes diagnosticados de EC entre los años 1986 y 2006, todos ellos mayores de 18 años, y seguidos en las consultas externas de nuestro hospital. Los criterios utilizados en el diagnóstico de EC fueron:

1. Paciente con clínica compatible o sugerente de EC.

2. Biopsia duodenal o yeyunal con atrofia de las vellosidades.

3. Respuesta clínica y/o histológica tras dieta sin gluten.

Se tomaron al menos cuatro biopsias duodenales en el momento del diagnóstico a todos los pacientes mediante endoscopia digestiva alta. El grado de atrofia se clasificó según la clasificación de Marsh (IIIa, IIIb, IIIc) (2). Tras instaurar una dieta sin gluten se comprobó mejoría clínica en todos los pacientes. La mejoría histológica se comprobó en los 11 pacientes que contaban con segunda biopsia duodenal.

Hemos recogido datos referidos a los síntomas de presentación, enfermedades asociadas, bioquímica, serología (anticuerpos antigliadina y AEm) y genética. Los pacientes han sido clasificados en función de si padecían o no manifestaciones digestivas, en concreto diarrea, coincidiendo con algún dato analítico de malabsorción. El $87,1 \%$ de los pacientes contaban con estudio de anticuerpos antiendomisio (AEm) mediante inmunofluorescencia indirecta sobre esófago de mono en el momento del diagnóstico y el 80,6 y 83,8\% de los pacientes con anticuerpos antigliadina de tipo IgA e IgG respectivamente. La presencia de los alelos DQ2 (DQA1*0501 y DQ B1*02) se ha estudiado en el 61,3\% (19/31) de los pacientes mediante SSP-PCR (PCR específica de alelo). Hemos comprobado si la clínica típica o la presencia de AEm se asocian a diferencias en cuanto a la expresión clínica, serología o grado de atrofia de las vellosidades. Los valores se expresan como mediana y rango. Las pruebas esta-

dísticas empleadas han sido, para la edad el test de U Mann Whitney y para las variables cualitativas la prueba de Chi cuadrado.

\section{RESULTADOS}

La mediana de edad del grupo fue de 41 años, predominando las mujeres (21 pacientes, 67,7\%). El 51,6\% de los pacientes presentaba diarrea. La tabla I muestra las diferencias entre los pacientes con y sin diarrea. El 16,1\% (5/31) de los pacientes tenía dolor abdominal pero no diarrea y el 32,2\% (10/31) de los pacientes no presentaba diarrea o dolor abdominal que, en principio, sugiriesen una enfermedad intestinal, entre ellos los tres pacientes con diagnóstico previo de dermatitis herpetiforme. Los antecedentes médicos, los síntomas de los pacientes y los datos de laboratorio se recogen en las tablas II y III.

Tabla I. Diferencias encontradas entre los grupos con y sin manifestaciones digestivas

\begin{tabular}{lccc}
\hline & Con diarrea (16) & Sin diarrea (15) & $p$ \\
\hline Edad (mediana y rango) & 47 & 36 & NS \\
& $20-63$ & $20-61$ & \\
Mujeres & $68,7 \%(11 / 16)$ & $66,7 \%(10 / 15)$ & NS \\
Hombres & $31,3 \%(5 / 16)$ & $33,3 \%(5 / 15)$ & \\
Serología & & & \\
AEm+ & $64,3 \%(9 / 14)$ & $76,9 \%(10 / 13)$ & NS \\
AEm- & $35,7 \%(5 / 14)$ & $23,1 \%(3 / 13)$ & \\
Atrofia vellosidades & & & \\
Marsh III a & $12,5 \%(2 / 16)$ & $46,7 \%(7 / 15)$ & 0,03 \\
Marsh III b-c & $87,5 \%(14 / 16)$ & $53,3 \%(8 / 15)$ & \\
HLA DQ2 & & & \\
Dos alelos & $60 \%(6 / 10)$ & $77,8 \%(7 / 9)$ & \\
Un alelo & $30 \%(3 / 10)$ & $0 \%$ & NS \\
Ningún alelo & $10 \%(1 / 10)$ & $22,2 \%(2 / 9)$ & \\
\hline
\end{tabular}

Tabla II. Enfermedades asociadas al diagnóstico de EC

\begin{tabular}{lc}
\hline Trastornos ginecológicos & \\
(aborto, infertilidad) & $33,3 \%(*)$ \\
Trastornos ansioso-depresivos & $19,4 \%$ \\
Hipotiroidismo & $12,9 \%$ \\
Dermatitis herpetiforme & $9,7 \%$ \\
Crisis comiciales & $3,2 \%$ \\
\hline (*) Del total de mujeres
\end{tabular}

Tabla III. Manifestaciones clínicas y de laboratorio de los pacientes con diagnóstico de EC

Dolor abdominal

Diarrea o esteatorrea

Anemia ferropénica

Déficit de vitamina $B_{12}$

Alargamiento del tiempo de protrombina

Déficit de folatos

Hipoproteinemia

Elevación de GOT y/o GPT
$54,8 \%(17 / 31)$

$51,6 \%(16 / 31)$

$45,2 \%(14 / 31)$

$50 \%(6 / 12)$

$42,9 \%(3 / 7)$

$41,7 \%(5 / 12)$

$25 \%(4 / 16)$

$9,7 \%(3 / 31)$ 
En lo referente al grado de atrofia el 29\% (9 pacientes) presentaba atrofia vellositaria parcial (grado IIIa de Marsh), el 32,2\% (10 pacientes) presentaba atrofia subtotal (grado IIIb de Marsh) y el 38,7\% restante (12 pacientes) presentaba atrofia vellositaria total (grado IIIc de Marsh). Los AEm fueron positivos en el 70,4\% (19/27) de los pacientes que contaban con estudio serológico. Los anticuerpos antigliadina de tipo $\operatorname{IgA}$ se detectaron en el $40 \%$ de los 25 pacientes que tenían realizada esta determinación y los de tipo $\operatorname{IgG}$ en el $57,7 \%$ de 26 pacientes. Ninguno de los pacientes estudiados con serología tenía deficiencia de IgA. En el estudio genético, el 68,4\% (13/19) era portador de los dos alelos estudiados, el $15,8 \%$ (3/19) era portador de uno de los alelos y el restante $15,8 \%$ no era portador de ninguno de los dos alelos. En la tabla IV se describen las diferencias encontradas entre pacientes con AEm positivos y negativos.

Tabla IV. Diferencias relacionadas con la positividad/negatividad de AEm

\begin{tabular}{lccc}
\hline & AEm positivos (19) & AEm negativos (8) & $p$ \\
\hline Edad (mediana y rango) & 37 & 40 & NS \\
& $22-59$ & $20-59$ & \\
Mujeres & $78,9 \%(15 / 19)$ & $37,5 \%(3 / 8)$ & 0,03 \\
Hombres & $21,1 \%(4 / 19)$ & $62,5 \%(5 / 8)$ & \\
Clínica & & & \\
Típica & $47,4 \%(9 / 19)$ & $62,5 \%(5 / 8)$ & NS \\
Atípica & $52,6 \%(10 / 19)$ & $37,5 \%(3 / 8)$ & \\
Atrofia vellosidades & & & \\
Marsh Illa & $15,8 \%(3 / 19)$ & $50 \%(4 / 8)$ & 0,05 \\
Marsh Illb-c & $84,2 \%(16 / 19)$ & $50 \%(4 / 8)$ & \\
HLA DQ2 & & & \\
$\quad$ Dos alelos & $70 \%(7 / 10)$ & $66,7 \%(4 / 6)$ & \\
Un alelo & $10 \%(1 / 10)$ & $16,7 \%(1 / 6)$ & NS \\
Ningún alelo & $20 \%(2 / 10)$ & $16,7 \%(1 / 6)$ & \\
\hline
\end{tabular}

\section{DISCUSIÓN}

La EC ha sido clásicamente considerada una enfermedad de la infancia sin embargo hasta un $20 \%$ de los adultos son diagnosticados con más de 60 años (3). Tanto en países del Norte de Europa como en el nuestro la prevalencia descrita de EC es muy elevada, entre 1 caso por cada 100 a 389 personas (4-7). Nuestro hospital atiende una población rural y urbana de unos 210.000 habitantes, por tanto, aunque en nuestro medio el mayor número de diagnósticos se hace en la infancia, la serie de pacientes adultos que presentamos refleja que aún estamos lejos de la prevalencia descrita y que la inmensa mayoría de casos aún no está diagnosticada (3). Por otro lado el que no hayamos considerado celiacos a pacientes sin atrofia de las vellosidades (grados I y II de Marsh) y el que no hayamos hecho una búsqueda de celiacos entre familiares de los pacientes pueden explicar en parte un pequeño número de diagnósticos. A lo largo de los años los diagnósticos han aumentado (fig. 1) probablemente por un mejor co-

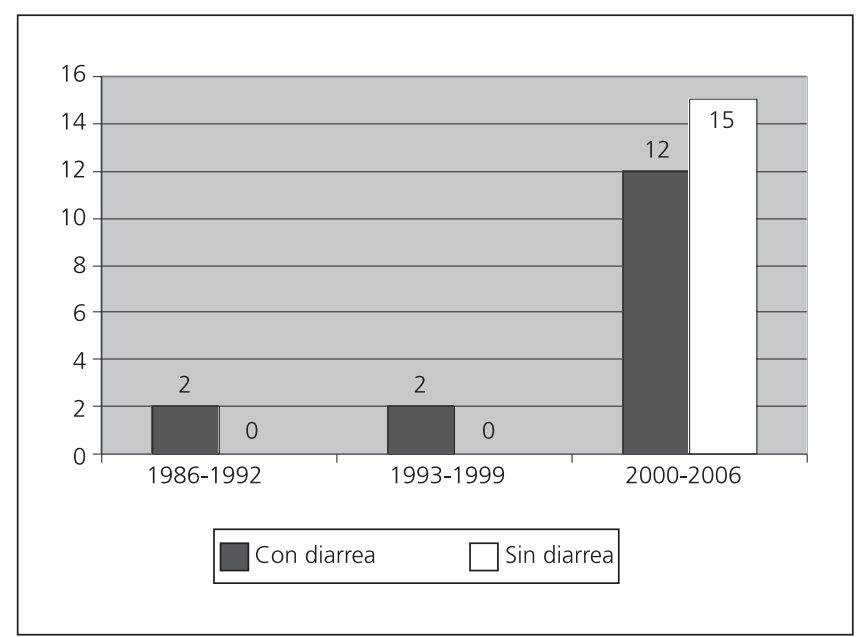

Fig. 1. Número de diagnósticos de enfermedad celíaca del adulto desde 1986 hasta 2006.

nocimiento de la enfermedad que sin embargo aún parece insuficiente. Probablemente la concienciación entre profesionales y los protocolos de prevención secundaria como el que se está implantando en la comunidad de Madrid pueden ayudar a remediar este problema.

Las enfermedades asociadas a la EC, las manifestaciones clínicas de los pacientes que describimos y la proporción de pacientes sin clínica digestiva son similares a las que otros autores comunican (8-13). Como otros, vemos que la proporción de pacientes sin diarrea e incluso con estreñimiento ha aumentado en los últimos años $(3,9,10)$. Es relevante la frecuencia con la que encontramos dolor abdominal como único síntoma entre nuestros pacientes. También detectamos deficiencia de vitamina B12 con relativa frecuencia, ya descrita por otros (14), y mayor aún en nuestra serie que la de ácido fólico. La asociación descrita de EC con hipertransaminasemia de causa desconocida, con hepatopatías autoinmunes o de otro tipo debe hacer incluir la serología de la EC en el estudio de las hipertransaminasemias y de algunas hepatopatías (15-19). En cualquier caso la positividad de AEm o anticuerpos antitransglutaminasa tisular (ATGt) debe seguirse de la confirmación histológica de la EC $(20,21)$.

La detección de los ATGt con ELISA es preferible a la detección con imunofluorescencia de los AEm por ser más sencilla y objetiva. La deteción de ATGt se ha introducido en nuestro hospital en el último año y por eso la serología empleada en el diagnóstico de los pacientes de nuestra serie han sido los AEm. En la serie que describimos la frecuencia con la que se detectan AEm ha sido del $70 \%$, inferior a la publicada por algunos autores $(13,22)$ pero similar a la descrita por otros $(14,23)$. Diferencias de método entre los laboratorios (24), la interpretación subjetiva de la inmunofluorescencia indirecta o que la población infantil y la adulta no sean comparables en este aspecto pueden explicar estas diferencias. Se ha descrito la asociación entre la presencia de AEm y ATGt y manifes- 
taciones clínicas en pacientes sin diagnóstico previo de EC (25) mientras que por otro lado la negatividad de los AEm no es rara en el diagnóstico en adultos y puede asociarse a formas más avanzadas de la EC $(13,26)$. Igualmente se ha demostrado que la sensibilidad de la serología varía en función de la intensidad de la atrofia intestinal $(13,23,27)$. Nuestros resultados apoyan esta posibilidad al encontrar mayor atrofia con más frecuencia en pacientes con serología positiva. De la misma manera pensamos que los diferentes grados de atrofia de vellosidades descritos por Marsh (2), aún teniendo en cuenta que en la EC la lesión de la mucosa es parcheada, pudieran corresponderse con formas clínicas más o menos graves. En nuestros pacientes también encontramos la clínica digestiva asociada a mayor atrofia de vellosidades. Sin embargo, se ha demostrado por otros autores que el grado de atrofia de las vellosidades no se relaciona con la forma de presentación $(23,28)$ y que los pacientes con enteropatía por gluten con Marsh I (infiltrado linfocitario y sin atrofia) pueden tener tanta expresión clínica como los pacientes con atrofia de las vellosidades (29). Esto necesariamente modifica los criterios diagnósticos de la EC en la práctica clínica dejando de ser necesaria la atrofia de vellosidades para el diagnóstico de la EC y requiriéndose por parte de los patólogos un estudio y descripción del infiltrado linfoide de la mucosa intestinal (3).

La frecuencia con la que detectamos el alelo DQ2 también es algo inferior a lo publicado $(30,31)$. La presencia de los DQ2 ó DQ8 es considerada una condición necesaria pero no suficiente para el desarrollo de la EC (12,32). En pacientes con clínica digestiva clara encontramos uno o dos alelos en el $90 \%$ de los pacientes y entre pacientes sin diarrea encontramos uno o dos alelos en el 77,7\%. El $10 \%$ de los pacientes con serología positiva porta un único alelo y el $20 \%$ no porta ninguno de los dos. Puede tratarse de portadores del alelo DQ8 que no ha sido estudiado, o que otros genes descritos estén presentes en estos pacientes. Moléculas de tipo HLA-I concretamente MICA y MICB han sido recientemente asociados a la EC (33). El estudio genético se ha incorporado a las diferentes vías propuestas para el diagnóstico de la EC de forma que trabajos recientes apoyan la detección de HLA DQ2 y DQ8 para el screening y la toma de biopsias en caso de positividad $(29,34,35)$. Esta opción apoyaría el diagnóstico en el 66,6\% de nuestros pacientes sin AEm al ser portadores de los dos alelos. Nuestra serie es muy pequeña como para comparar la utilidad de la serología y el estudio genético en el diagnóstico de la EC. En cualquier caso la frecuencia de AEm en nuestros pacientes nos obliga a no dejar de hacer la gastroscopia aunque la serología sea negativa o a hacer previamente un estudio genético antes de optar por no biopsiar al paciente. De no ser así dejaríamos de diagnosticar el 35\% de los pacientes con diarrea y al $23 \%$ de los pacientes sin diarrea.

Debe estudiarse la vía más segura y rentable en nuestro medio para llegar al diagnóstico de la EC como han hecho otros autores en pacientes con clínica compatible y en los diferentes grupos de riesgo (familiares de celiacos, pacientes con síndrome de Down, Turner u otros) $(32,35,36)$. Una posibilidad puede ser tomar biopsias de duodeno en el transcurso de una endoscopia solicitada a pacientes con dispepsia, flatulencia u otros síntomas y sobre todo si se trata de pacientes con anemia sin causa clara, coexisten enfermedades autoinmunes o hay elevación de las transaminasas (37). En cualquier caso el diagnóstico puede ser difícil (pocos síntomas, serología negativa, biopsia de duodeno con cambios mínimos) pero debe ser firme dadas las exigencias y la dificultad de seguir una dieta sin gluten $(3,11)$ por eso el seguimiento de los pacientes repitiendo los test serológicos, una dieta sin gluten de prueba, una segunda biopsia intestinal que demuestre mejoría de la histología con la dieta, la sobrecarga oral de gluten en casos con resultados histológicos poco claros $(12,13,38)$ y quizá un estudio más profundo de la biopsia intestinal, como exploraciones inmunocitométricas de las poblaciones linfoides de la mucosa (39) o el depósito de anticuerpos antitransglutaminasa de tipo 2 en la mucosa duodenal (26) son herramientas que habrá que tener presentes.

\section{BIBLIOGRAFÍA}

1. León AJ, Garrote JA, Arranz E. Citocinas en la patogenia de la enfermedad celiaca. Med Clin (Barc) 2005; 125: 508-16.

2. Marsh MN. Gluten, major histocompatibility complex and the small intestine: A molecular and immunologic approach to the spectrum of gluten-sensitive (celiac sprue). Gastroenterology 1992; 102: 330-54.

3. Rodrigo Sáez L. La enfermedad celiaca en el adulto. Rev Esp Enferm Dig 2006: 98: 397-407.

4. García-Novo MD, Garfia C, Acuña Quirós, Asensio J, Zancada G, Barrio Gutiérrez S, et al. Prevalencia de la enfermedad celiaca en donantes de sangre de la Comunidad de Madrid. Rev Esp Enferm Dig 2007; 99: 337-42.

5. Dube C, Rostom A, Sy R, Craney A, Saloojee N, Garritty C, et al. The prevalence of coeliac disease in average-risk and at-risk Western European populations: A systematic review. Gastroenterology 2005; 128: S57-67.

6. Hovdenak N, Hovlid E, Askens L, Fluge G, Erichsen MM, Eide J. High prevalence of asymptomatic coeliac disease in Norway: study of blood donors. Eur J Gastroenerol Hepatol 1999; 11: 185-7.

7. Riestra S, Fernández E, Rodrigo L, García S, Ocio G. Prevalence of coeliac disease in the general population of the northern of Spain. Strategies of serologic screening. Scand J Gastroenterol 2003; 35: 398-402.

8. Green PHR, Stavropoulos SN, Panagi SG, Goldstein SL, Mcmahon DJ, Absan H, et al. Characteristics of adult celiac disease in the USA: Results of a national survey. Am J Gastroenterol 2001; 96: 126-31.

9. Tursi A, Giorgetti G, Brandimarte G, Rubino E, Lombardi D, Gasbarrini G. Prevalence and clinical presentation of subclinical/silent celiac disease in adults: An analysis on a 12-year observation. Hepatogastroenterology 2001; 48: 462-4.

10. Bottaro G, Cataldo F, Rotolo N, Spina M, Corazza GR. The clinical pattern of subclinical/silent celiac disease: an analysis on 1026 consecutive cases. Am J Gastroenterol 1999; 94: 691-6.

11. Casellas F, López Vivancos J, Malagelada JR. Epidemiología actual y accesibilidad al seguimiento de la dieta de la enfermedad celiaca del adulto. Rev Esp Enferm Dig 2006; 98: 408-19.

12. Rodrigo L. Celiac disease. World J Gastroenterol 2007; 12: 6585-93.

13. Hopper AD, Hadjivassiliou M, Butt S, Sanders DS. Adult celiac disease. Br Med J 2007; 335: 558-62. 
14. Dahele A, Ghosh S. Vitamin B12 deficiency in untreated celiac disease. Am J Gastroenterol 2001; 96: 745-50.

15. Bardella MT, Valenti L, Pagliari C, Perocchi M, Fare M, Fracanzani AL, et al. Searching for celiac disease in patients with non-alcoholic fatty liver disease. Dig Liver Dis 2004; 36: 333-6.

16. Volta U, Granito A, De Franceschi L, Petrolini N, Bianchi FB. Anti tissue transglutaminase antibodies as predictors of silent celiac disease in patients with hypertransaminasaemia of unknown origin. Dig Liver Dis 2001; 33: 420-5.

17. Múgica F, Aranzadi MJ, Recasens M, Almagro F, Muñagorri A, Elósegui E, et al. Enfermedad celiaca del adulto e hipertransaminasemia. Rev Esp Enferm Dig 2000; 92: 78-81.

18. Villalta D, Girolami D, Bidoli E, Bizzaro N, Tampoia M, Liguori M, et al. High prevalence of celiac disease in autoimmune hepatitis detected by anti-tissue transglutaminase autoantibodies. J Clin Lab Anal 2005; 19: 6-10.

19. Pazo M, Abdulkader I, Otero-Antón E, Tomé S, Villamil I, González Quintela A. Alteraciones hepáticas en la enfermedad celiaca del adulto. Caracterización clínico-patológica y evolución. Gastroenterol Hepatol 2006; 29: 383-9.

20. Lo Iacono O, Petta S, Venezia G, DiMarco V, Tarantino G, Barbaria $\mathrm{F}$, et al. Anti-tissue transglutaminase antibodies in patients with abnormal liver tests: Is it always celiac disease? Am J Gastroenterol 2005; 100: 2471-7.

21. Germenis AE, Yiannaki EE, Zachou K, Roka V, Barbanis S, Liaskos $\mathrm{C}$, et al. Prevalence and clinical significance of immunoglobulin A antibodies against tissue transglutaminase in patients with diverse chronic liver diseases. Clin Diagn Lab Immunol 2005; 12: 941-8.

22. Lerner A, Kumar V, Iancu TC. Immunological diagnosis of childhood coeliac disease: Comparison between antigliadin, antireticulin and antiendomysial antibodies. Clin Exp Immunol 1994; 95: 78-82.

23. Abrams JA, Diamond B, Rotterdam H, Green PH. Seronegative celiac disease: Increased prevalence with lesser degrees of villous atrophy. Dig Dis Sci 2004; 49: 546-50.

24. AGA Technical review on celiac sprue. Gastroenterology 2001; 120: 1526-40.

25. West J, Logan RF, Hill PG, Khaw KT. The iceberg of celiac disease: What is below the waterline? Clin Gastroenterol Hepatol 2007; 5: 5962 .

26. Salmi TT, Collin P, Korponay-Szabo IR, Laurila K, Partanen J, Huhtala $\mathrm{H}$, et al. Endomysial antibody-negative coeliac disease: Clinical characteristics and intestinal autoantibody deposits. Gut 2006, 55:
1746-53.

27. Rostom A, Dubé C, Cranney A, Cranney A, Saloojee N, Sy R, et al The diagnostic accuracy of serologic tests for celiac disease: A systematic review. Gastroenterology 2005: 128: S38-46.

28. Brar P, Kwon GY, Egbuna II, Holleran S, Ramakrishnan R, Bhagat G, et al. Lack of correlation of degree of villous atrophy with severity of clinical presentation of celiac disease. Dig Liver Dis 2007; 39: 26-9.

29. Esteve M, Rosinach M, Fernández-Bañares F, Farre C, Salas A, Alsina $M$, et al. Spectrum of gluten sensitive enteropathy in first degree relatives of coeliac patients: Clinical relevance of lymphocytic enteritis. Gut 2006; 55: 1739-45.

30. Van Heel DA. Genetics in celiac disease. Best Pract Res Clin Gastroenterol 2005; 19: 323-9.

31. Karell K, Louka AS, Moodie SJ, et al. HLA types in celiac disease patients not carrying the DQA1*05-DQB1*02 (DQ2) heterodimer: Results from the European genetics cluster on celiac disease. Hum Immunol 2003; 64: 469-77.

32. Green PHR. Where are all those patients with celiac disease? Am J Gastroenterol 2007; 102: 1461-3.

33. López-Vázquez A, Rodrigo L, Fuentes D, Riestra S, Bousoño C, García-Fernández S, et al. MHC class I chain related gene A (MICA) modulates the development of celiac disease in patients with the high risk heterodimer DQA $1 * 0501 / \mathrm{DQB} 1 * 0201$. Gut 2002; 50: 336-40.

34. Fernández-Bañares F, Esteve M, Alsina M, et al. Systematic evaluation of the causes of chronic watery diarrhea with functional characteristics. Gastroenterology 2006; 130 (4 Supl. 2): A 318.

35. Dorn SD, Matchar DB. Cost-effectiveness analysis of strategies for diagnosing celiac disease. Dig Dis Sci 2007 (En prensa).

36. Catassi C, Kryszak D, Louis-Jacques O, Duerksen DR, Hill I, Crowe $\mathrm{SE}$, et al. Detection of celiac disease in primary care: A multicente case-finding study in North America. Am J Gastroenterol 2007; 102: 1454-60.

37. Riestra S, Domínguez F, Fernández-Ruiz E, García-Riesco E, Nieto $\mathrm{R}$, Fernández E, et al. Usefulness of duodenal biopsy during routine upper gastrointestinal endoscopy for diagnosis of celiac disease. World J Gastroenterol 2006; 12: 5028-32.

38. Wahab PJ, Crusius JBA, Meijer JWR, Mulder CJJ. Gluten challenge in borderline gluten-sensitive enteropathy. Am J Gastroenterol 2001; 96: 1464-9

39. León F, Eiras P, Roy G, Camarero C. Intestinal intraepithelial lymphocytes and anti-transglutaminase in a screening algorithm for coeliac disease. Gut 2000; 47: 628-31 